\title{
An SDP Method for Copositivity of Partially Symmetric Tensors
}

\author{
Chunyan Wang, ${ }^{1}$ Haibin Chen $\mathbb{D}^{1},{ }^{1}$ and Haitao Che $\mathbb{D}^{2}$ \\ ${ }^{1}$ School of Management Science, Qufu Normal University, Rizhao, Shandong, China \\ ${ }^{2}$ School of Mathematics and Information Science, Weifang University, Weifang, Shandong, China \\ Correspondence should be addressed to Haibin Chen; chenhaibin508@qfnu.edu.cn
}

Received 1 July 2020; Accepted 24 July 2020; Published 18 August 2020

Guest Editor: Chuanjun Chen

Copyright (c) 2020 Chunyan Wang et al. This is an open access article distributed under the Creative Commons Attribution License, which permits unrestricted use, distribution, and reproduction in any medium, provided the original work is properly cited.

\begin{abstract}
In this paper, we consider the problem of detecting the copositivity of partially symmetric rectangular tensors. We first propose a semidefinite relaxation algorithm for detecting the copositivity of partially symmetric rectangular tensors. Then, the convergence of the proposed algorithm is given, and it shows that we can always catch the copositivity of given partially symmetric tensors. Several preliminary numerical results confirm our theoretical findings.
\end{abstract}

\section{Introduction}

Let $\mathscr{A}=\left(a_{i_{1} i_{2}, \ldots, i_{p} j_{1} j_{2}, \ldots, j_{q}}\right)$ be a real $(p, q)$-th order $m \times n$-dimensional rectangular tensor, where $a_{i_{1} i_{2}, \ldots, i_{p} j_{1} j_{2}, \ldots, j_{q}} \in \mathbb{R}$ for $i_{k} \in[m], k \in[p], j_{l} \in[n]$, and $l \in[q]$. If the entries of the tensor are invariant under any permutation of $i_{1}, i_{2}, \ldots, i_{p}$ and $j_{1}, j_{2}, \ldots, j_{q}, \mathscr{A}$ is called a partially symmetric tensor. For the sake of simplicity, let $\mathbb{P} \mathbb{S}_{p, q}^{m \times n}$ be the set of all partially symmetric rectangular tensors with order $(p, q)$ and dimension $m \times n$. By the relationship between partially symmetric tensors and homogeneous polynomials, we always use the following notation:

$$
f(\mathbf{x}, \mathbf{y})=\mathscr{A} \mathbf{x}^{p} \mathbf{y}^{q}=\sum_{\substack{i_{1}, \ldots, i_{p} \in[m] \\ j_{1}, \ldots, j_{q} \in[n]}} a_{i_{1} i_{2}, \ldots, i_{p} j_{1} j_{2}, \ldots, j_{q}} x_{i_{1}} x_{i_{2}}, \ldots, x_{i_{p}} y_{j_{1}} y_{j_{2}}, \ldots, y_{j_{q}}
$$

By this notation, we know that $\mathscr{A}=\left(a_{i_{1}, \ldots, i_{p} j_{1}, \ldots, j_{q}}\right) \in \mathbb{P S}_{p, q}^{m \times n}$ is strictly copositive if and only if

$\mathscr{A} \mathbf{x}^{p} \mathbf{y}^{q} \geq(>) 0, \quad$ for all $\mathbf{x} \in \mathbb{R}_{+}^{m}, \mathbf{y} \in \mathbb{R}_{+}^{n}$ with $\|\mathbf{x}\|=1,\|\mathbf{y}\|=1$.

Particularly, if $m=n$ and $\mathbf{x}=\mathbf{y}$, then it reduces to the copositivity of symmetric tensors [1-10].
The copositive tensor has attracted many researches' attention since it plays an important role in polynomial optimization [11], hypergraph theory [1], vacuum stability of a general scalar potential [12], tensor complementarity problem [13, 14], tensor eigenvalue complementarity problem [15, 16], and so on [17-37]. Kannike proved the vacuum stability conditions for more complicated potentials with the help of the copositive tensor [12]. Ling et al. [16] proposed that the tensor generalized eigenvalue complementarity problem is solvable and has one solution at least under assumptions that the related square tensor is strictly copositive. During the process of application, a challenging problem is how to detect the copositivity of tensors numerically.

Recently, several numerical algorithms are proposed to check the copositivity of symmetric tensors. To the best of our knowledge, the first numerical algorithm was proposed by Chen et al. in [2], where the algorithm is based on the representation of the multivariate form in barycentric coordinates with respect to the standard simplex. Then, by a suitable convex subcone of a copositive tensor cone, an updated numerical algorithm for copositivity of tensors was proposed in [1]. It must be pointed out that the methods of $[1,2]$ can only capture strictly copositive tensors and noncopositive tensors. To overcome this drawback, in [38], $\mathrm{Li}$ et al. proposed an SDP relaxation algorithm to test the 
copositivity of higher-order tensors. Very recently, Nie et al. gave a complete semidefinite relaxation algorithm for detecting the copositivity of a matrix or tensor [39]. If the potential tensor is copositive, the algorithm can get a certificate for the copositivity. Otherwise, the algorithm can get a point that refutes the copositivity. Furthermore, it is showed that the detection can be done by solving a finite number of semidefinite relaxations for all matrices and tensors.

For the copositivity of partially symmetric tensors, Gu et al. gave the first two spectral properties in [40], and some necessary or sufficient conditions for a real partially symmetric rectangular tensor to be copositive are further established. Moreover, an equivalent notion of strictly copositive rectangular tensors is presented [40]. In [41], Wang et al. extended the simplicial partition method for symmetric tensors to check the copositivity of partially symmetric tensors. However, as we discussed above, it can only capture all strictly copositive rectangular tensors or noncopositive rectangular tensors. When the input tensor is copositive but not strictly copositive, the algorithm may not stop in general. To solve this, motivated by the algorithm of $[38,39]$, we propose a new algorithm to check the copositivity of partially symmetric tensors in this paper.

The remainder of this paper is organized as follows. In Section 2, we recall some preliminaries on polynomials. In Section 3, we formulate the potential problem as a proper polynomial optimization problem which can be efficiently solved by Lasserre-type semidefinite relaxations. Then, a numerical method is proposed to check whether a given partially symmetric tensor is copositive or not, and the convergence of this algorithm is established. Several numerical experiments are listed in Section 4, and final remarks are given in Section 5.

\section{Preliminaries}

Let $\mathbb{R}[\mathbf{x}]$ be the ring of the polynomial with variables $\mathbf{x}=\left(x_{1}, x_{2}, \ldots, x_{n}\right) \in \mathbb{R}^{n}$. Let $\mathbb{R}[\mathbf{x}]_{d} \subseteq \mathbb{R}[\mathbf{x}]$ denote the vector space of polynomials with degree at most $d$. The degree of a polynomial $f$ is denoted as deg $(f)$. Denote e as the vector of all entries which equals one. A polynomial $p$ is called SOS if there exist $p_{1}, p_{2}, \ldots, p_{r} \in \mathbb{R}[\mathbf{x}]$ such that $p=p_{1}^{2}+p_{2}^{2}+, \ldots,+p_{r}^{2}$. Denote by $\sum[\mathbf{x}]$ the set of all SOS polynomials. For $\mathbf{x} \in \mathbb{R}^{n}$ and $\alpha \in N^{n}$, let $\mathbf{x}^{\alpha}=x_{1}^{\alpha_{1}} x_{2}^{\alpha_{2}}, \ldots, x_{n}^{\alpha_{n}}$. Then, for any polynomial $f \in \mathbb{R}[\mathbf{x}]$, it can be denoted by $f(\mathbf{x})=\sum_{\alpha \in N^{n}} f_{\alpha} \mathbf{x}^{\alpha}$, and $\operatorname{vec}(f):=$ $\left(f_{\alpha}\right)_{\alpha \in \mathbb{N}^{n}}$ denotes its sequence of coefficients in the monomial basis of $\mathbb{R}[\mathbf{x}]$. For matrix $A$, its transpose is denoted by $A^{\top}$. For a symmetric matrix $X, X \geqslant 0$ means $X$ is positive semidefinite. More details about polynomial optimization can be seen in [42-45].

For $\alpha=\left(\alpha_{1}, \alpha_{2}, \ldots, \alpha_{n}\right) \in N^{n},|\alpha|=\alpha_{1}+, \cdots,+\alpha_{n}$, and denote $N_{d}^{n}=\left\{\alpha \in N^{n}|| \alpha \mid \leq d\right\}$. For $t \in \mathbb{R},\lceil t\rceil$ denotes the smallest integer that is not smaller than $t$. If the subset $I \subseteq \mathbb{R}[\mathbf{x}]$ satisfies that $I+I \subseteq I$ and $I \cdot \mathbb{R}[\mathbf{x}] \subseteq \mathbb{R}[\mathbf{x}]$, then $I$ is called ideal. For a polynomial tuple $h=\left(h_{1}, h_{2}, \ldots, h_{s}\right)$, the ideal generated by $h$ is defined such that

$$
\mathscr{I}(h)=h_{1} \mathbb{R}[\mathbf{x}]+h_{2} \mathbb{R}[\mathbf{x}]+, \ldots,+h_{s} \mathbb{R}[\mathbf{x}] .
$$

The $k$-th truncation ideal generated by $h$ is

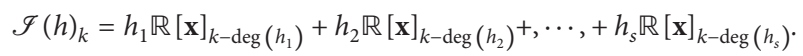

For complex and real algebraic varieties of polynomial tuple $h$, define

$$
\left\{\begin{array}{l}
V_{C}(h)=\left\{\mathbf{x} \in C^{n} \mid h(\mathbf{x})=0\right\}, \\
V_{R}(h)=V_{C}(h) \cap \mathbb{R}^{n} .
\end{array}\right.
$$

The quadratic module generated by $g=\left(g_{1}, g_{2}, \ldots, g_{t}\right)$ is (denote $g_{0}=1$ )

$$
Q(g)_{k}=\sum[\mathbf{x}]+g_{1} \sum[\mathbf{x}]+, \ldots,+g_{t} \sum[\mathbf{x}] .
$$

For $\mathbf{y}=\left(y_{\alpha}\right) \in \mathbb{R}^{N_{d}^{n}}, \alpha \in N_{d}^{n}$, where $\mathbb{R}^{N_{d}^{n}}$ is the space of real vectors indexed by $\alpha \in N_{d}^{n}$, define

$$
\left\langle\sum_{\alpha \in N_{d}^{n}} p_{\alpha} x_{1}^{\alpha_{1}} x_{2}^{\alpha_{2}}, \ldots, x_{n}^{\alpha_{n}}, \mathbf{y}\right\rangle=\sum_{\alpha \in N_{d}^{n}} p_{\alpha} y_{\alpha} .
$$

For a polynomial $q \in \mathbb{R}[\mathbf{x}]_{2 k}$, the $k$-th localizing matrix of $q$ is the symmetric matrix $L_{k}^{q}(\mathbf{y})$ satisfying

$$
\operatorname{vec}\left(p_{1}\right)^{\top}\left(L_{q}^{(k)}(\mathbf{y})\right) \operatorname{vec}\left(p_{2}\right)=\left\langle q p_{1} p_{2}, \mathbf{y}\right\rangle
$$

for all $p_{1}, p_{2} \in \mathbb{R}[\mathbf{x}]$ with $\operatorname{deg}\left(p_{1}\right), \operatorname{deg}\left(p_{2}\right) \leq k-\lceil\operatorname{deg}(q) / 2\rceil$, where $\operatorname{vec}\left(p_{i}\right)$ denotes the coefficient vector of the polynomial $p_{i}$. When $q=1, L_{q}^{(k)}(\mathbf{y})$ is the moment matrix $M_{k}(\mathbf{y})=L_{1}^{(k)}(\mathbf{y})$. Let $f=\left(f_{1}, f_{2}, \ldots, f_{r}\right)$ be a polynomial tuple; its localizing matrix is defined such that

$$
L_{f}^{(k)}(\mathbf{y})=\left(L_{f_{1}}^{(k)}(\mathbf{y}), L_{f_{2}}^{(k)}(\mathbf{y}), \ldots, L_{f_{r}}^{(k)}(\mathbf{y})\right)
$$

\section{The SDP Algorithm for Copositivity of Partially Symmetric Tensors}

In this section, we establish an equivalent condition for the copositivity of partially symmetric tensors. Then, the concerned problem can be reformulated as a polynomial optimization problem. To continue, recall that a partially symmetric tensor $\mathscr{A} \in \mathbb{P} \mathbb{S}_{p, q}^{m \times n}$ is strictly copositive if and only if

$$
\mathscr{A} \mathbf{x}^{p} \mathbf{z}^{q} \geq 0(>0), \quad \text { for all } \mathbf{x} \in \mathbb{R}_{+}^{m}, \mathbf{z} \in \mathbb{R}_{+}^{n} \text { with }\|\mathbf{x}\|=1,\|\mathbf{z}\|=1,
$$

which is equivalent with the following optimization problem:

$$
\begin{aligned}
f^{*}=\min & \mathscr{A} \mathbf{x}^{p} \mathbf{z}^{q} \\
\text { s.t. } & \mathbf{e}_{1}^{\top} \mathbf{x}=1, \mathbf{e}_{2}^{\top} \mathbf{z}=1 \\
& \mathbf{x} \in \mathbb{R}_{+}^{m}, \mathbf{z} \in \mathbb{R}_{+}^{n} .
\end{aligned}
$$

Clearly, tensor $\mathscr{A}$ is strictly copositive if and only if $f^{*} \geq 0(>0)$. Problem (11) can be solved by classical Lasserre relaxations [46]. Since the objection function is continuous and the feasible region is compact, problem (11) always has a 
solution. Without loss of generality, assume $\left(\mathbf{x}^{*}, \mathbf{z}^{*}\right)$ is one of the solutions of (11); then, it satisfies the following KKTconditions with $\lambda, \mu \in \mathbb{R}, \mathbf{v} \in \mathbb{R}^{m}$, and $\mathbf{w} \in \mathbb{R}^{n}$ :

$$
\left\{\begin{array}{l}
p \mathscr{A} \mathbf{x}^{* p^{-1}} \mathbf{z}^{* q}-\lambda \mathbf{e}_{1}-\mathbf{v}=\mathbf{0}, \\
q \mathscr{A} \mathbf{x}^{* p} \mathbf{z}^{* q-1}-\mu \mathbf{e}_{2}-\mathbf{w}=\mathbf{0}, \\
\mathbf{e}_{1}^{\top} \mathbf{x}^{*}=1, \mathbf{e}_{2}^{\top} \mathbf{z}^{*}=1, \\
\mathbf{x}^{*} \geq \mathbf{0}, \mathbf{z}^{*} \geq \mathbf{0}, \mathbf{v} \geq \mathbf{0}, \mathbf{w} \geq \mathbf{0}, \\
\mathbf{x}^{* \top} \mathbf{v}=0, \mathbf{z}^{* \top} \mathbf{w}=0
\end{array}\right.
$$
and

By (12), we obtain that $\lambda=p \mathscr{A} \mathbf{x}^{*{ }^{p}} \mathbf{z}^{* q}, \mu=q \mathscr{A} \mathbf{x}^{* p} \mathbf{z}^{* q}$,

$$
\begin{aligned}
& p \mathscr{A} \mathbf{x}^{* p-1} \mathbf{z}^{q}-\lambda \mathbf{e}_{1} \geq \mathbf{0}, q \mathscr{A} \mathbf{x}^{* p} \mathbf{z}^{* q-1}-\mu \mathbf{e}_{2} \geq \mathbf{0}, \\
& \mathbf{x}^{* \top}\left(p \mathscr{A} \mathbf{x}^{* p-1} \mathbf{z}^{* q}\right)-\lambda \mathbf{x}^{* \top} \mathbf{e}_{1}=0, \mathbf{z}^{* \top}\left(q \mathscr{A} \mathbf{x}^{* p} \mathbf{z}^{* q-1}\right)-\mu \mathbf{z}^{* \top} \mathbf{e}_{2}=0 .
\end{aligned}
$$

Combining this with the fact that $\left\|\mathbf{x}^{*}\right\| \leq 1,\left\|\mathbf{z}^{*}\right\| \leq 1$, we consider the following optimization problem:

$$
\begin{array}{ll}
\min & \mathscr{A} \mathbf{x}^{p} \mathbf{z}^{q} \\
\text { s.t. } & \mathbf{x}^{\top}\left(\mathscr{A} \mathbf{x}^{p-1} \mathbf{z}^{q}\right)-\left(\mathscr{A} \mathbf{x}^{p} \mathbf{z}^{q}\right) \mathbf{x}^{\top} \mathbf{e}_{1}=0, \\
& \mathbf{z}^{\top}\left(\mathscr{A} \mathbf{x}^{p} \mathbf{z}^{q-1}\right)-\left(\mathscr{A} \mathbf{x}^{p} \mathbf{z}^{q}\right) \mathbf{z}^{\top}=0, \\
& \mathscr{A} \mathbf{x}^{p-1} \mathbf{z}^{q}-\left(\mathscr{A} \mathbf{x}^{p} \mathbf{z}^{q}\right) \mathbf{e}_{1} \geq \mathbf{0}, \\
& \mathscr{A} \mathbf{x}^{p} \mathbf{z}^{q-1}-\left(\mathscr{A} \mathbf{x}^{p} \mathbf{z}^{q}\right) \mathbf{e}_{2} \geq \mathbf{0}, \\
& \mathbf{e}_{1}^{\top} \mathbf{x}=1, \mathbf{e}_{2}^{\top} \mathbf{z}=1,1-\|\mathbf{x}\|^{2} \geq 0,1-\|\mathbf{z}\|^{2} \geq 0 \\
& \mathbf{x} \in \mathbb{R}_{+}^{m}, \mathbf{z} \in \mathbb{R}_{+}^{n} .
\end{array}
$$

It is clear to see that problems (11) and (14) are equivalent in the sense that they have the same optimal solution. To solve (14), we introduce the following notations:

$$
\left\{\begin{array}{l}
f(\mathbf{x}, \mathbf{z})=\mathscr{A} \mathbf{x}^{p} \mathbf{z}^{q}, \\
g(\mathbf{x}, \mathbf{z})=\left\{\mathscr{A} \mathbf{x}^{p-1} \mathbf{z}^{q}-\left(\mathscr{A} \mathbf{x}^{p} \mathbf{z}^{q}\right) \mathbf{e}_{1}, \mathscr{A} \mathbf{x}^{p} \mathbf{z}^{q-1}-\left(\mathscr{A} \mathbf{x}^{p} \mathbf{z}^{q}\right) \mathbf{e}_{2}, 1-\|\mathbf{x}\|^{2}, 1-\|\mathbf{z}\|^{2}, x_{i}, z_{j}\right\}, \\
h(\mathbf{x}, \mathbf{z})=\left\{\mathbf{e}^{\top}(\mathbf{x}, \mathbf{0})_{n+m}=1, \mathbf{e}^{\top}(\mathbf{0}, \mathbf{z})_{n+m}=1, x_{i}\left(\mathscr{A} \mathbf{x}^{p-1} \mathbf{z}^{q}\right)_{i}-\left(\mathscr{A} \mathbf{x}^{p} \mathbf{z}^{q}\right) x_{i}, z_{j}\left(\mathscr{A} \mathbf{x}^{p} \mathbf{z}^{q-1}\right)_{j}-\left(\mathscr{A} \mathbf{x}^{p} \mathbf{z}^{q}\right) z_{j}\right\} .
\end{array}\right.
$$

So, the problem of (14) can be rewritten such that

$$
\begin{aligned}
f^{*}=\min & f(\mathbf{x}, \mathbf{z}) \\
\text { s.t. } & g(\mathbf{x}, \mathbf{z}) \geq \mathbf{0}, \\
& h(\mathbf{x}, \mathbf{z})=\mathbf{0} .
\end{aligned}
$$

By the Lasserre-type semidefinite relaxations of (16), consider the semidefinite program

$$
\begin{aligned}
\rho_{k}=\min & \sum_{\alpha \in N^{n+m}} f_{\alpha} y_{\alpha} \\
\text { s.t. } & L_{g}^{(k)}(\mathbf{y}) \geqslant \mathbf{0}, L_{h}^{(k)}(\mathbf{y})=\mathbf{0}, \\
& y_{0}=1, M_{k}(\mathbf{y}) \geqslant \mathbf{0}, \mathbf{y} \in \mathbb{R}^{N_{2 k}^{n+m},}
\end{aligned}
$$

where $k=k_{0}, k_{0}+1, \ldots$, with $k_{0}=\max \{\lceil(p / 2)\rceil,\lceil(q / 2)\rceil\}$. It is obvious that the feasible set is compact, and the Archimedean condition holds. Thus, the asymptotic convergence of (17) is always guaranteed. Moreover, $\mathscr{A}$ is copositive if $\rho_{k} \geq 0$ for some $k$, and $\rho_{k}$ is a monotonically decreasing sequence, with the decreasing of order $k$, i.e.,

$$
\rho_{k_{0}} \leq \rho_{k_{0}+1} \leq, \ldots, \leq \rho_{k} \leq, \ldots, \leq f^{*} .
$$

Now, we present an algorithm to check the copositivity of a given partially symmetric rectangular tensor (Algorithm 1).

$$
\begin{aligned}
\rho_{k}^{*}=\min & \left\langle\xi^{\top}[x, y]_{m+n}, \mathbf{y}\right\rangle \\
\text { s.t. } & y_{0}=1, L_{g}^{(k)}(\mathbf{y}) \geqslant \mathbf{0}, L_{h}^{(k)}(\mathbf{y})=\mathbf{0} \\
& M_{k}(\mathbf{y}) \geqslant \mathbf{0}, L_{\rho_{k}-f(\mathbf{x}, \mathbf{z})}^{(k)} \geqslant \mathbf{0}, \mathbf{y} \in \mathbb{R}^{N_{2 k}^{n+m}},
\end{aligned}
$$

The following theorem shows the convergence of $\mathrm{Al}$ gorithm 1 for any partially symmetric tensor.

Theorem 1. Suppose $\mathscr{A} \in \mathbb{P S}_{p, q}^{m \times n}$ is a partially symmetric tensor. Then, the following properties hold:

(i) For all $k \geq 0$, problem (16) is feasible and achieves its optimal value $\rho_{k}=f^{*}$ for all $k$ sufficiently large

(ii) For all $k \geq 0$, problem (19) has an optimizer if it is feasible

(iii) If $\mathscr{A}$ is copositive, Algorithm 1 must stop with $\rho^{k} \geq 0$ when $k$ is sufficiently large

(iv) If $\mathscr{A}$ is not copositive, Algorithm 1 must stop with $f(x, z)<0$ for almost all $\xi \in \mathbb{R}^{\mathbb{N}_{p+q}^{n+m}}$ when $k$ is sufficiently large

Proof

(i) Since the feasible set of (11) is compact, it must have a minimizer $\left(\mathbf{x}^{*}, \mathbf{z}^{*}\right)$. On the contrary, $\left(\mathbf{x}^{*}, \mathbf{z}^{*}\right)$ is a feasible point for (16), which implies that the semidefinite relaxation (17) is always feasible. Since $L_{1-\|x\|^{2}}^{(k)} \geqslant 0$, let $X=\{(\mathbf{x}, 0)$, $\left.(0, \mathbf{z}) \mid \mathbf{x} \in \mathbb{R}^{m}, \mathbf{z} \in \mathbb{R}^{n}\right\} \subseteq \mathbb{R}^{m+n}$; then, it holds that $L_{1-\|\mathrm{x}\|^{2}}^{(k)} \geqslant 0$. We now show that the feasible set of $(17)$ is compact as follows. First of all, we have

$$
1 \geq y_{2 e_{1}}+y_{2 e_{2}}+, \ldots,+y_{2 e_{n+m}}
$$


Step 0: given an arbitrary vector $\xi \in \mathbb{R}^{\mathbb{N} \mathbb{N}_{p+q}^{n+m}}$. Let $k=\max \{[(p / 2)],[(q / 2)]\}$.

Step 1: solve the semidefinite relaxation (17). If $\rho_{k} \geq 0$, then stop, and $\mathscr{A}$ is copositive. If $\rho_{k}<0$, go to Step 2.

Step 2: solve the following semidefinite program:

for an optimizer $\mathbf{y}^{*}$ if it is feasible. If it is infeasible, let $k=k+1$ and go to Step 1.

Step 3: let $\left(\mathbf{x}^{*}, \mathbf{z}^{*}\right)=\left(\left(y^{*}\right)_{e_{1}}, \ldots,\left(y^{*}\right)_{e_{m}},\left(y^{*}\right)_{e_{m+1}}, \ldots,\left(y^{*}\right)_{e_{m+n}}\right)$. If $\mathscr{A} \mathbf{x}^{* p^{*}} \mathbf{z}^{* q}<0$, then $\mathscr{A}$ is not copositive and stop. Otherwise, let $k=k+1$ and go to Step 1 .

Algorithm 1: An SDP method for copositivity of a partially symmetric tensor $\mathscr{A} \in \mathbb{P} \mathbb{S}_{p, q}^{m \times n}$.

Then, $0 \leq y_{2 e_{i}} \leq 1$; since $M_{k}(\mathbf{y}) \geqslant 0, i \in[m+n]$. Furthermore, for all $0<|\alpha| \leq k-1$, the $(\alpha, \alpha)$-th diagonal entry of $L_{1-\|\mathbf{x}\|^{2}}^{(k)}$ is nonnegative, which implies that

$$
y_{2 \alpha} \geq y_{2 e_{1}+2 \alpha}+y_{2 e_{2}+2 \alpha}+\ldots,+y_{2 e_{n+m}+2 \alpha}
$$

Take $\alpha=e_{1}, e_{2}, \ldots, e_{m+n}$ in the following analysis. By the same argument as (21) and repeating $k-1$ times, we can show that $0 \leq y_{2 \beta} \leq 1$ for all $|\beta| \leq k$. By the definition of $M_{k}(\mathbf{y})$, we know that the diagonal entries $M_{k}(\mathbf{y})$ are precisely $y_{2 \beta},|\beta| \leq k$. Since $M_{k}(\mathbf{y}) \geqslant 0$, all the entries of $M_{k}(\mathbf{y})$ must be between -1 and 1 . So, $\mathbf{y}$ is bounded, and the feasible set of (17) is compact. Hence, the optimal value can always be achieved. In the following, we will show that $\rho_{k}=f^{*}$ for all $k$ sufficiently large.
By direct computation, the optimization (16) is equivalent with the following problem:

$$
\begin{array}{ll}
\min & \mathscr{A} \mathbf{x}^{p} \mathbf{z}^{q} \\
\text { s.t. } & x_{i}\left(\mathscr{A} \mathbf{x}^{p-1} \mathbf{z}^{q}\right)_{i}-\left(\mathscr{A} \mathbf{x}^{p} \mathbf{z}^{q}\right) x_{i}=0, \\
& z_{j}\left(\mathscr{A} \mathbf{x}^{p} \mathbf{z}^{q-1}\right)_{j}-\left(\mathscr{A} \mathbf{x}^{p} \mathbf{z}^{q}\right) z_{j}=0, \\
& \mathscr{A} \mathbf{x}^{p-1} \mathbf{z}^{q}-\left(\mathscr{A} \mathbf{x}^{p} \mathbf{z}^{q}\right) \mathbf{e}_{1} \geq \mathbf{0}, \\
& \mathscr{A} \mathbf{x}^{p} \mathbf{z}^{q-1}-\left(\mathscr{A} \mathbf{x}^{p} \mathbf{z}^{q}\right) \mathbf{e}_{2} \geq \mathbf{0}, \\
& \mathbf{e}_{1}^{\top} \mathbf{x}=1, \mathbf{e}_{2}^{\top} \mathbf{z}=1 \\
& \mathbf{x} \in \mathbb{R}_{+}^{m}, \mathbf{z} \in \mathbb{R}_{+}^{n} .
\end{array}
$$

For simplicity, denote

$$
\left\{\begin{array}{l}
f(\mathbf{x}, \mathbf{z})=\mathscr{A} \mathbf{x}^{p} \mathbf{z}^{q}, \\
g(\mathbf{x}, \mathbf{z})=\left\{(\mathbf{x}, \mathbf{z}), \mathscr{A} \mathbf{x}^{p-1} \mathbf{z}^{q}-\left(\mathscr{A} \mathbf{x}^{p} \mathbf{z}^{q}\right) \mathbf{e}_{1}, \mathscr{A} \mathbf{x}^{p} \mathbf{z}^{q-1}-\left(\mathscr{A} \mathbf{x}^{p} \mathbf{z}^{q}\right) \mathbf{e}_{2}\right\} \\
h(\mathbf{x}, \mathbf{z})=\left\{\mathbf{e}^{\top}(\mathbf{x}, \mathbf{0})_{m+n}-1, \mathbf{e}^{\top}(\mathbf{0}, \mathbf{z})_{m+n}-1, x_{i}\left(\mathscr{A} \mathbf{x}^{p-1} \mathbf{z}^{q}\right)_{i}-\left(\mathscr{A} \mathbf{x}^{p} \mathbf{z}^{q}\right) x_{i}, z_{j}\left(\mathscr{A} \mathbf{x}^{p} \mathbf{z}^{q-1}\right)_{j}-\left(\mathscr{A} \mathbf{x}^{p} \mathbf{z}^{q}\right) z_{j}\right\} .
\end{array}\right.
$$

Corresponding Lasserre's relaxations for (22) are

$$
\begin{aligned}
\rho_{k}^{\prime}=\min & \sum_{\alpha \in N^{n}} f_{\alpha} y_{\alpha} \\
\text { s.t. } & L_{g}^{(k)}(\mathbf{y}) \geqslant \mathbf{0}, L_{h}^{(k)}(\mathbf{y})=\mathbf{0}, \\
& y_{0}=1, M_{k}(\mathbf{y}) \geqslant \mathbf{0}, \mathbf{y} \in \mathbb{R}^{N_{2 k}^{n+m}} .
\end{aligned}
$$

For

$$
k=k_{0}, k_{0}+1, \ldots,
$$

where

$k_{0}=\max \{[(p / 2)],[(q / 2)]\}$, any feasible solution of

(17) is also a feasible solution of (24), so

$$
\rho_{k}^{\prime} \leq \rho_{k} \leq f^{*}, \quad k=k_{0}, k_{0}+1, \ldots
$$

Next, we show that the set of polynomials

$$
F=\left\{\left(1-\mathbf{e}^{T} \mathbf{x}\right) \phi+\sum_{i=1}^{n} x_{i}\left(\sum_{l} s_{l}^{2}\right)+\left(1-\mathbf{e}^{T} \mathbf{z}\right) \psi+\sum_{i=1}^{n} z_{j}\left(\sum_{t} s_{t}^{2}\right)\right\} .
$$

is Archimedean, i.e., there exists $f \in F$ such that the inequality $f(\mathbf{x}) \geq 0$ defines a compact set in $\mathbb{R}^{m+n}$. Let $f=2-\|X\|^{2}$ and $X=(\mathbf{x}, \mathbf{z})_{m+n}$; we have

$$
\begin{aligned}
2-\|\mathbf{x}\|^{2}= & \left(1-\mathbf{e}^{T} \mathbf{x}\right)\left(1+\|\mathbf{x}\|^{2}\right)+\sum_{i=1}^{n} x_{i}\left(1-x_{i}\right)^{2} \\
& +\sum_{i \neq j=1}^{m} x_{i}^{2} x_{j}+\left(1-\mathbf{e}^{T} \mathbf{z}\right)\left(1+\|\mathbf{z}\|^{2}\right)+\sum_{j=1}^{n} z_{j}\left(1-z_{j}\right)^{2} \\
& +\sum_{i \neq j=1}^{n} z_{i}^{2} z_{j} .
\end{aligned}
$$


So, $F$ is Archimedean by Theorem 3.3 of [47]; we know that $\rho_{k}^{\prime}=f^{*}$ when $k$ is sufficiently large. Hence, $\rho_{k}=f^{*}$ when all $k$ values are sufficiently large.

(ii) The proof is the same with (i).

(iii) Clearly, $\mathscr{A}$ is copositive if and only if $f^{*} \geq 0$. By item (i), $\rho_{k}=f^{*}$ for all $k$ big enough. Therefore, if $\mathscr{A}$ is copositive, we must have $\rho_{k} \geq 0$ for all $k$ large enough.

(iv) If $\mathscr{A}$ is not copositive, then $f^{*}<0$. By (i), there exists $k_{1} \in N$ such that $\rho_{k}=f^{*}$ for all $k \geq k_{1}$. Hence, for all $k \geq k_{1}$, problem (19) is equivalent with the following problem:

$$
\begin{aligned}
\widehat{\rho_{k}}=\min & \left\langle\xi^{\top}[\mathbf{x}, \mathbf{y}]_{m+n}, \mathbf{y}\right\rangle \\
\text { s.t. } & y_{0}=1, L_{1-\mathbf{e}^{T} \mathbf{x}}^{(k)}(\mathbf{y}) \geqslant \mathbf{0}, L_{1-\mathbf{e}^{T} \mathbf{y}}^{(k)} \geqslant \mathbf{0}, L_{X}^{(k)}(\mathbf{y}) \geqslant \mathbf{0}, \\
& M_{k}(\mathbf{y}) \geqslant \mathbf{0}, L_{f^{*}-f(X)}^{(k)} \geqslant \mathbf{0}, X=(\mathbf{x}, \mathbf{z})_{m+n}, \mathbf{y} \in \mathbb{R}^{N_{2 k}^{n+m}} .
\end{aligned}
$$

It is $k$-th Lasserre's relaxation for the polynomial optimization

$$
\begin{array}{ll}
\min & \xi^{\top}[\mathbf{x}, \mathbf{z}]_{m+n} \\
\text { s.t. } & 1-\mathbf{e}^{\top}(\mathbf{x}, \mathbf{0})_{m+n} \geq 0,1-\mathbf{e}^{\top}(\mathbf{0}, \mathbf{z})_{m+n} \geq 0, \mathbf{x} \geq 0, \mathbf{z} \geq 0, f^{*}-f(X) \geq 0 .
\end{array}
$$

The feasible region of (29) is clearly compact. When $\xi \in \mathbb{R}^{\mathbb{N}_{p+q}^{n+m}}$ is arbitrary, (29) has a unique optimizer $X^{*}=\left(\mathbf{x}^{*}, \mathbf{z}^{*}\right)$. Hence, for almost all $\xi \in \mathbb{R}^{\mathbb{N}_{p+q}^{n+m}}, X^{*}$ is the unique optimizer. For notation convenience, denote by $\widehat{\mathbf{y}}^{k}$ the optimizer of (19) with the relaxation order $k$. Let $X^{k}=\left(\left(\widehat{\mathbf{y}}^{k}\right)_{e_{1}}, \ldots,\left(\widehat{\mathbf{y}}^{k}\right)_{\mathbf{e}_{n+m}}\right)$. By Corollary 3.5 of [48] or Theorem 3.3 of [49], the sequence $\left\{X^{k}\right\}_{k=k_{0}}^{\infty}$ must converge to $X^{*}$. Since $f^{*} \leq \rho_{k}^{*}<0$, we must have $f\left(X_{k}\right)<0$ when $k$ is sufficiently large.

\section{Numerical Examples}

In this section, we give several numerical examples to show the efficiency of Algorithm 1. Let $S_{\pi\left(i_{1} i_{2}, \ldots, i_{m}\right)}$ denote the set of all permutations of $i_{1} i_{2}, \ldots, i_{m}$, and let $\rho_{k}^{*}=0$ when $\left|\rho_{k}^{*}\right|<1 e-5$. All experiments are done in Matlab2014b on a desktop computer with Intel (R) Core (TM)i7-6500 CPU @ $2.50 \mathrm{GHz} 2.60 \mathrm{GHz}$ and $16 \mathrm{~GB}$ of RAM.

Example 1. Suppose that $\mathscr{A} \in \mathbb{P} \mathbb{S}_{2,2}^{2 \times 4}$ is given by

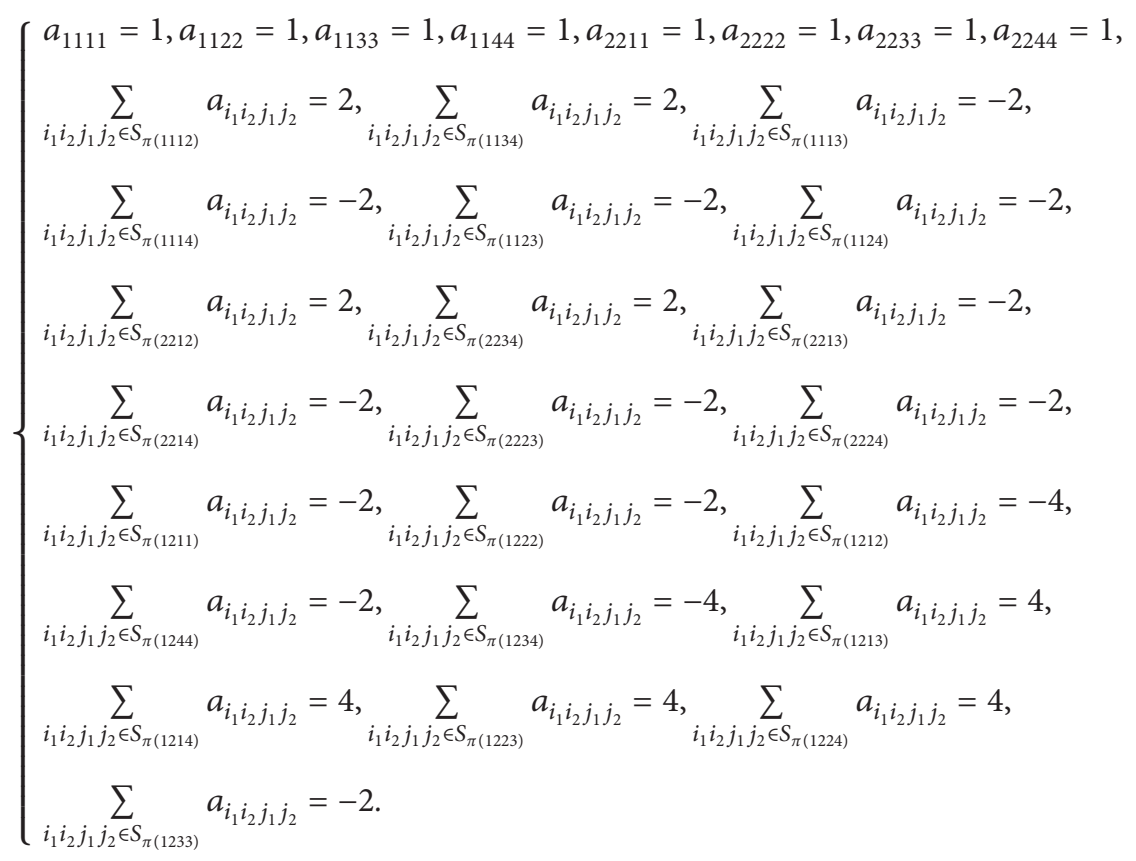

The corresponding polynomial for tensor $\mathscr{A}$ is

$$
\begin{aligned}
f(\mathbf{x}, \mathbf{y}) & =\left(x_{1}-x_{2}\right)^{2}\left(y_{1}+y_{2}-y_{3}-y_{4}\right)^{2}, \mathbf{x} \\
& =\left(x_{1}, x_{2}\right), \mathbf{y}=\left(y_{1}, y_{2}, y_{3}, y_{4}\right) .
\end{aligned}
$$

By Algorithm 1, we know that $f^{*}=0$ with $\mathbf{x}=(0.5000,0.5000), \quad \mathbf{y}=(0.2500,0.2500,0.2500,0.2500)$, which implies that rectangular tensor $\mathscr{A}$ is copositive.
Example 2. Suppose that $\mathscr{A} \in \mathbb{P S}_{2,2}^{1 \times 2}$ with entries such that

$$
\left\{\begin{array}{l}
a_{1111}=1, \\
a_{1122}=1, \\
\sum_{i_{1} i_{2} j_{1} j_{2} \in S_{\pi(11,12)}} a_{i_{1} i_{2} j_{1} j_{2}}=-2 .
\end{array}\right.
$$

The corresponding polynomial of $\mathscr{A}$ is 


$$
f(\mathbf{x}, \mathbf{y})=x_{1}^{2} y_{1}^{2}-2 x_{1}^{2} y_{1} y_{2}+x_{1}^{2} y_{2}^{2}, \mathbf{x}=\left(x_{1}\right), \mathbf{y}=\left(y_{1}, y_{2}\right)
$$

By Algorithm 1, we obtain that $f^{*}=0$ with optimal solution $(\mathbf{x}, \mathbf{y})=(1.0000,0.7071,0.7071)$, which implies that $\mathscr{A}$ is copositive but not strictly copositive.

Example 3. Suppose that $\mathscr{A} \in \mathbb{P} \mathbb{S}_{2,2}^{2 \times 2}$ is given by

$$
\left\{\begin{array}{l}
a_{1111}=1, \\
a_{1122}=1, \\
a_{2211}=1, \\
a_{2222}=1, \\
\sum_{i_{1} i_{2} j_{1} j_{2} \in S_{\pi(11,12)}} a_{i_{1} i_{2} j_{1} j_{2}}=-2, \\
\sum_{i_{1} i_{2} j_{1} j_{2} \in S_{\pi(22,12)}} a_{i_{1} i_{2} j_{1} j_{2}}=-2, \\
\sum_{i_{1} i_{2} j_{1} j_{2} \in S_{\pi(12,11)}} a_{i_{1} i_{2} j_{1} j_{2}}=2, \\
\sum_{i_{1} i_{2} j_{1} j_{2} \in S_{\pi(12,22)}} a_{i_{1} i_{2} j_{1} j_{2}}=2, \\
\sum_{i_{1} i_{2} j_{1} j_{2} \in S_{\pi(12,12)}} a_{i_{1} i_{2} j_{1} j_{2}}=-4 .
\end{array}\right.
$$

So, the corresponding polynomial of $\mathscr{A}$ is that

$$
\begin{aligned}
f(\mathbf{x}, \mathbf{y})= & x_{1}^{2} y_{1}^{2}+x_{1}^{2} y_{2}^{2}-2 x_{1}^{2} y_{1} y_{2}+x_{2}^{2} y_{1}^{2}+x_{2}^{2} y_{2}^{2} \\
& -2 x_{2}^{2} y_{1} y_{2}+2 x_{1} x_{2} y_{1}^{2}+2 x_{1} x_{2} y_{2}^{2}-4 x_{1} x_{2} y_{1} y_{2}
\end{aligned}
$$

where $\mathbf{x}=\left(x_{1}, x_{2}\right), \mathbf{y}=\left(y_{1}, y_{2}\right)$. By Algorithm 1, we have $f^{*}=0 \quad$ with $\mathbf{x}^{*}=(0.5126,0.4874), \mathbf{y}^{*}=(0.5000, .5000)$, which implies that the rectangular tensor is copositive.

Example 4. Suppose that $\mathscr{A} \in \mathbb{P S}_{3,2}^{3 \times 2}$ is given by

$$
\left\{\begin{array}{l}
a_{11122}=1, \\
a_{22222}=1, \\
a_{33311}=1, \\
\sum_{i_{1} i_{2} i_{3} j_{1} j_{2} \in S_{\pi(123,12)}} a_{i_{1} i_{2} i_{3} j_{1} j_{2}}=-3 .
\end{array}\right.
$$

The corresponding polynomial of the partially symmetric rectangular tensor $\mathscr{A}$ is

$$
f(\mathbf{x}, \mathbf{y})=x_{1}^{3} y_{2}^{2}+x_{2}^{3} y_{2}^{2}+x_{3}^{3} y_{1}^{2}-4 x_{1} x_{2} x_{3} y_{1} y_{2},
$$

where $\mathbf{x}=\left(x_{1}, x_{2}, x_{3}\right), \mathbf{y}=\left(y_{1}, y_{2}\right)$. By Algorithm 1, we know that $f^{*}=-0.0639$ with $\mathbf{x}^{*}=(0.7652,0.4702,0.7652)$, $\mathbf{y}^{*}=(0.3572,0.8724)$, which implies that the rectangular tensor is not copositive.

Example 5. Suppose $\mathscr{A} \in \mathbb{P S}_{2,2}^{2 \times 2}$ is a tensor with entries such that

$$
\left\{\begin{array}{l}
a_{1111}=1, \\
a_{1122}=-1, \\
a_{2211}=1, \\
a_{2222}=1, \\
\sum_{i_{1} i_{2} j_{1} j_{2} \in S_{\pi(11,12)}} a_{i_{1} i_{2} j_{1} j_{2}}=2, \\
\sum_{i_{1} i_{2} j_{1} j_{2} \in S_{\pi(22,12)}} a_{i_{1} i_{2} j_{1} j_{2}}=2, \\
\sum_{i_{1} i_{2} j_{1} j_{2} \in S_{\pi(12,11)}} a_{i_{1} i_{2} j_{1} j_{2}}=2, \\
\sum_{i_{1} i_{2} j_{1} j_{2} \in S_{\pi(12,22)}} a_{i_{1} i_{2} j_{1} j_{2}}=2, \\
\sum_{i_{1} i_{2} j_{1} j_{2} \in S_{\pi(12,12)}} a_{i_{1} i_{2} j_{1} j_{2}}=-4 .
\end{array}\right.
$$

The corresponding polynomial of the partially symmetric rectangular tensor $\mathscr{A}$ is

$$
\begin{aligned}
f(\mathbf{x}, \mathbf{y})= & x_{1}^{2} y_{1}^{2}-x_{1}^{2} y_{2}^{2}+2 x_{1}^{2} y_{1} y_{2}+x_{2}^{2} y_{1}^{2}+x_{2}^{2} y_{2}^{2} \\
& +2 x_{2}^{2} y_{1} y_{2}+2 x_{1} x_{2} y_{1}^{2}+2 x_{1} x_{2} y_{2}^{2}-4 x_{1} x_{2} y_{1} y_{2}
\end{aligned}
$$

where $\mathbf{x}=\left(x_{1}, x_{2}\right), \mathbf{y}=\left(y_{1}, y_{2}\right)$. By Algorithm 1 , we know that $f^{*}=0.3333$ with $\mathbf{x}^{*}=(0.6666,0.3334)$, $\mathbf{y}^{*}=(0.5000, .5000)$, which implies that the rectangular tensor is strictly copositive.

\section{Conclusions}

In this paper, based on Lasserre's hierarchy of semidefinite relaxations, we propose a new criterion to judge whether a given partially symmetric rectangular tensor is copositive or not. The convergence for the proposed algorithm is established. Furthermore, numerical examples demonstrate that the proposed algorithm is effective when the input rectangular tensor has lower dimension and orders, and it is difficult for the case with higher order or higher dimension. We will continue to study this problem in the future.

\section{Data Availability}

The data used to support the findings of this study are available from the corresponding author upon request.

\section{Conflicts of Interest}

The authors declare that there are no conflicts of interest regarding the publication of this paper.

\section{Authors' Contributions}

Each author contributed equally to this paper and read and approved the final manuscript.

\section{Acknowledgments}

This project was supported by the Natural Science Foundation of China (11601261), the Shandong Provincial 
Natural Science Foundation (ZR2019MA022), and Project of Shandong Province Higher Educational Science and Technology Program (Grant no. J14LI52).

\section{References}

[1] H. Chen, Z.-H. Huang, and L. Qi, "Copositive tensor detection and its applications in physics and hypergraphs," Computational Optimization and Applications, vol. 69, no. 1, pp. 133-158, 2018.

[2] H. Chen, Z.-H. Huang, and L. Qi, "Copositivity detection of tensors: theory and algorithm," Journal of Optimization Theory and Applications, vol. 174, no. 3, pp. 746-761, 2017.

[3] L. Qi, "Eigenvalues of a real supersymmetric tensor," Journal of Symbolic Computation, vol. 40, no. 6, pp. 1302-1324, 2005.

[4] L. Qi, "Symmetric nonnegative tensors and copositive tensors," Linear Algebra and Its Applications, vol. 439, no. 1, pp. 228-238, 2013.

[5] Y. Wang, L. Caccetta, and G. Zhou, "Convergence analysis of a block improvement method for polynomial optimization over unit spheres," Numerical Linear Algebra with Applications, vol. 22, no. 6, pp. 1059-1076, 2015.

[6] Y. Wang, W. Liu, L. Caccetta, and G. Zhou, "Parameter selection for nonnegative 11 matrix/tensor sparse decomposition," Operations Research Letters, vol. 43, no. 4, pp. 423-426, 2015.

[7] Y. Wang, K. Zhang, and H. Sun, "Criteria for strong H-tensors," Frontiers of Mathematics in China, vol. 11, no. 3, pp. 577-592, 2016.

[8] K. Zhang and Y. Wang, "An H-tensor based iterative scheme for identifying the positive definiteness of multivariate homogeneous forms," Journal of Computational and Applied Mathematics, vol. 305, pp. 1-10, 2016.

[9] K. Zhang, H. Chen, H. Chen, and P. Zhao, "A potential reduction method for tensor complementarity problems," Journal of Industrial \& Management Optimization, vol. 15, no. 2, pp. 429-443, 2019.

[10] G. Zhou, G. Wang, L. Qi, and M. Alqahtani, "A fast algorithm for the spectral radii of weakly reducible nonnegative tensors," Numerical Linear Algebra with Applications, vol. 25, no. 2, p. e2134, 2018.

[11] J. Peña, J. C. Vera, and L. F. Zuluaga, "Completely positive reformulations for polynomial optimization," Mathematical Programming, vol. 151, no. 2, pp. 405-431, 2014.

[12] K. Kannike, "Vacuum stability of a general scalar potential of a few fields," European Physical Journal C, vol. 76, p. 324, 2016.

[13] H. Chen, L. Qi, and Y. Song, "Column sufficient tensors and tensor complementarity problems," Frontiers of Mathematics in China, vol. 13, no. 2, pp. 255-276, 2018.

[14] H. Chen and Y. Wang, "On computing minimal H-eigenvalue of sign-structured tensors," Frontiers of Mathematics in China, vol. 12, no. 6, pp. 1289-1302, 2017.

[15] J. Fan, J. Nie, and A. Zhou, "Tensor eigenvalue complementarity problems," Mathematical Programming, vol. 170, no. 2, pp. 507-539, 2018.

[16] C. Ling, H. He, and L. Qi, "On the cone eigenvalue complementarity problem for higher-order tensors," Computational Optimization and Applications, vol. 63, no. 1 , pp. 143-168, 2016.

[17] C. Chen, X. Zhang, G. Zhang, and Y. Zhang, "A two-grid finite element method for nonlinear parabolic integro-differential equations," International Journal of Computer Mathematics, vol. 96, no. 10, pp. 2010-2023, 2019.
[18] C. Chen, W. Liu, and C. Bi, "A two-grid characteristic finite volume element method for semilinear advection-dominated diffusion equations," Numerical Methods for Partial Differential Equations, vol. 29, no. 5, pp. 1543-1562, 2013.

[19] C. Chen and X. Zhao, "A posteriori error estimate for finite volume element method of the parabolic equations," $\mathrm{Nu}$ merical Methods for Partial Differential Equations, vol. 33, no. 1, pp. 259-275, 2017.

[20] C. Chen, K. Li, Y. Chen, and Y. Huang, "Two-grid finite element methods combined with Crank-Nicolson scheme for nonlinear Sobolev equations," Advances in Computational Mathematics, vol. 45, no. 2, pp. 611-630, 2019.

[21] C. Chen, H. Liu, X. Zheng et al., "A two-grid MMOC finite element method for nonlinear variable-order time-fractional mobile/immobile advectionCdiffusion equations," Computers and Mathematics with Applications, vol. 79, no. 9, pp. 27712783, 2019.

[22] H. Chen, L. Qi, L. Caccetta, and G. Zhou, "Birkhoff-von Neumann theorem and decomposition for doubly stochastic tensors," Linear Algebra and Its Applications, vol. 583, pp. 119-133, 2019.

[23] H. Chen, L. Qi, Y. Wang, and G. Zhou, "Further results on sum-of-squares tensors," Optimization Methods and Software, pp. 1-17, 2020.

[24] H. Chen, Y. Wang, and G. Zhou, "High-order sum-of-squares structured tensors: theory and applications," Frontiers of Mathematics in China, vol. 15, no. 2, pp. 255-284, 2020.

[25] M. Dong and H. Chen, "Geometry of the copositive tensor cone and its dual," Asia-Pacific Journal of Operational Researcch, vol. 380, 2020.

[26] J. He, X. Zhang, L. Liu, Y. Wu, and Y. Cui, “A singular fractional kelvin-voigt model involving a nonlinear operator and their convergence properties," Boundary Value Problems, vol. 2019, p. 112, 2019.

[27] J. He, X. Zhang, L. Liu, and Y. Wu, "Existence and nonexistence of radial solutions of the Dirichlet problem for a class of general k-Hessian equations," Nonlinear Analysis: Modelling and Control, vol. 23, no. 4, pp. 475-492, 2018.

[28] W. Wang, H. Chen, and Y. Wang, "A new C-eigenvalue interval for piezoelectric-type tensors," Applied Mathematics Letters, vol. 100, p. 106035, 2020.

[29] X. Zhang, L. Liu, and Y. Wu, "Multiple positive solutions of a singular fractional differential equation with negatively perturbed term," Mathematical and Computer Modelling, vol. 55, no. 3-4, pp. 1263-1274, 2012.

[30] X. Zhang, Y. Wu, and L. Caccetta, "Nonlocal fractional order differential equations with changing-sign singular perturbation," Applied Mathematical Modelling, vol. 39, no. 21, pp. 6543-6552, 2015.

[31] X. Zhang, L. Liu, Y. Wu, and Y. Cui, "A sufficient and necessary condition of existence of blow-up radial solutions for a $k$-Hessian equation with a nonlinear operator," Nonlinear Analysis: Modelling and Control, vol. 25, pp. 126-143, 2020.

[32] X. Zhang, J. Xu, J. Jiang, Y. Wu, and Y. Cui, “The convergence analysis and uniqueness of blow-up solutions for a Dirichlet problem of the general k-Hessian equations," Applied Mathematics Letters, vol. 102, p. 106124, 2020.

[33] X. Zhang, J. Jiang, Y. Wu, and Y. Cui, "The existence and nonexistence of entire large solutions for a quasilinear Schrödinger elliptic system by dual approach," Applied Mathematics Letters, vol. 100, p. 106018, 2020.

[34] X. Zhang, J. Jiang, Y. Wu, and Y. Cui, "Existence and asymptotic properties of solutions for a nonlinear Schrödinger 
elliptic equation from geophysical fluid flows," Applied Mathematics Letters, vol. 90, pp. 229-237, 2019.

[35] X. Zhang, L. Liu, Y. Wu, and Y. Cui, "The existence and nonexistence of entire large solutions for a quasilinear Schrödinger elliptic system by dual approach," Journal of Mathematical Analysis and Applications, vol. 464, no. 2, pp. 1089-1106, 2018.

[36] X. Zhang, L. Liu, Y. Wu, and Y. Cui, "Existence of infinitely solutions for a modified nonlinear Schrodinger equation via dual approach," Electron. J. Differential Equations, vol. 2147, pp. 1-15, 2018.

[37] X. Zhang, Y. Wu, and Y. Cui, "Existence and nonexistence of blow-up solutions for a Schrödinger equation involving a nonlinear operator," Applied Mathematics Letters, vol. 82, pp. 85-91, 2018.

[38] L. Li, X. Zhang, X. Zhang, Z.-H. Huang, and L. Qi, “Test of copositive tensors," Journal of Industrial \& Management Optimization, vol. 15, no. 2, pp. 881-891, 2019.

[39] J. Nie, Z. Yang, and X. Zhang, "A complete semidefinite algorithm for detecting copositive matrices and tensors," SIAM Journal on Optimization, vol. 28, no. 4, pp. 2902-2921, 2018.

[40] Y. Gu, W. Wu, and W. Wu, "Partially symmetric nonnegative rectangular tensors and copositive rectangular tensors," Journal of Industrial \& Management Optimization, vol. 15, no. 2, pp. 775-789, 2019.

[41] C. Wang, H. Chen, Y. Wang, and G. Zhou, "On copositiveness identification of partially symmetric rectangular tensors," Journal of Computational and Applied Mathematics, vol. 372, p. $112678,2020$.

[42] J. Lasserre, Moments, Positive Polynomials and Their Applications, Imperial College Press, London, UK, 2009.

[43] J. Lasserre, Introduction to Polynomial and Semi-algebraic Optimization, Cambridge University Press, Cambridge, UK, 2015.

[44] M. Laurent, "Optimization over polynomials: selected topics," in Proceedings of the International Congress of Mathematicians, Rio de Janeiro, Brazil, August 2014.

[45] M. Laurent, "Sums of squares, moment matrices and optimization over polynomials," Emerging Applications of Algebraic Geometry, vol. 149, pp. 157-270, 2009.

[46] J. B. Lasserre, "Global optimization with polynomials and the problem of moments," SIAM Journal on Optimization, vol. 11, no. 3, pp. 796-817, 2001.

[47] J. Nie, "Tight relaxations for polynomial optimization and Lagrange multiplier expressions," Mathematical Programming, vol. 178, no. 1-2, pp. 1-37, 2019.

[48] M. Schweighofer, "Optimization of polynomials on compact semialgebraic sets," SIAM Journal on Optimization, vol. 15, no. 3, pp. 805-825, 2005.

[49] J. Nie, "Polynomial optimization with real varieties," SIAM Journal On Optimization, vol. 23, no. 3, pp. 1634-1646, 2013. 